\title{
Prognostic significance of preoperative serum CA125, CA19-9 and CEA in gastric carcinoma
}

\author{
Wei Wang ${ }^{1,2, *}$, Xiao-Long Chen ${ }^{1,2, *}$, Shen-Yu Zhao ${ }^{3}$, Yu-Hui Xu ${ }^{3}$, Wei-Han Zhang ${ }^{1,2}$, \\ Kai Liu ${ }^{1,2}$, Xin-Zu Chen ${ }^{1,2}$, Kun Yang ${ }^{1,2}$, Bo Zhang ${ }^{1}$, Zhi-Xin Chen ${ }^{1}$, Jia-Ping Chen ${ }^{1}$, \\ Zong-Guang Zhou ${ }^{1,4}$ and Jian-Kun $\mathrm{Hu}^{1,2}$ \\ ${ }^{1}$ Department of Gastrointestinal Surgery, West China Hospital, Sichuan University, Chengdu, China \\ ${ }^{2}$ Laboratory of Gastric Cancer, State Key Laboratory of Biotherapy/Collaborative Innovation Center of Biotherapy and Cancer \\ Center, West China Hospital, Sichuan University, Chengdu, China \\ ${ }^{3}$ West China School of Medicine, Sichuan University, Chengdu, China \\ ${ }^{4}$ Laboratory of Digestive Surgery, State Key Laboratory of Biotherapy, West China Hospital, Sichuan University, Chengdu, \\ China \\ * These authors have contributed equally to this study and should be considered as co-first authors \\ Correspondence to: Jian-Kun Hu, email: hujkwch@126.com
}

Keywords: gastric cancer, tumor marker, CA125, CA19-9, CEA

Received: September 24, 2015 Accepted: April 05, 2016

Published: April 16, 2016

\section{ABSTRACT}

The prognostic significance of preoperative serum CA125, CA19-9 and CEA in gastric carcinoma (GC) has been widely reported and is still under debate. Here, we evaluated the prognostic significance of preoperative serum CA125, CA19-9 and CEA in patients with GC. 1692 patients with GC who underwent gastrectomy were divided into the training (from January 2005 to December 2011, $n=1024$ ) and the validation (from January 2012 to December 2013, $n=668$ ) cohorts. Positive groups of CA125 $(>13.72 \mathrm{U} / \mathrm{ml})$, CA19-9 $(>23.36 \mathrm{U} / \mathrm{ml})$ and CEA $(>4.28 \mathrm{ng} / \mathrm{ml})$ were significantly associated with more advanced clinicopathological traits and worse outcomes than that of negative groups (all $P<0.01)$. In Cox regression analysis, tumor size $(P<$ $0.001, P=0.005)$, pTNM stage $(P<0.001, P<0.001)$ and CA125 $(P=0.026, P=$ 0.005 ) were independent prognostic factors both in two cohorts. Nomograms of these two cohorts based on the number of positive serum tumor markers (NPTM) were more accurate in prognostic prediction than TNM stage alone. Our findings suggested that elevated preoperative serum CA125, CA19-9 and CEA were associated with more advanced clinicopathological traits and less favorable outcomes. In addition, CA125 as an independent prognostic factor should be further investigated. Nomogram based on NPTM could accurately predict the prognosis of GC patients.

\section{INTRODUCTION}

Gastric carcinoma (GC) is one of the most common malignant tumors with high mortality worldwide, especially in East Asia, and the five-year survival rate of GC is low [1-3]. Early diagnosis and treatment are the most efficient ways to improve the prognosis. Although examinations, like CT, ultrasound and gastroscopy, may provide much important information of tumor, it is still not sufficient to obtain complete data preoperatively. The detection of preoperative serum tumor markers is routinely used in a variety of tumor patients, since it may prompt the tumor burden and occult metastases of malignancies $[4,5]$. However, the clinical application of preoperative serum tumor markers is still under debate.

The commonly used tumor markers for GC patients in our hospital include carbohydrate antigen 125 (CA125), carbohydrate antigen 19-9 (CA19-9) and carcinoembryonic antigen (CEA). CA125 was first detected in ovarian cancer and had been reported to be positive in $80 \%$ of ovarian epithelial tumor patients [6]. In addition, the positivity of CA125 is also seen in breast cancer, pancreatic cancer, gastric cancer and some other tumors [7]. CA19-9 is a specific marker of 
digestive system tumors, especially pancreatic cancer, with high positive rate preoperatively and high negative rate postoperatively [8]. Thus, it is widely used in the diagnosis and monitoring of pancreatic cancer [9]. CEA, a non-specific tumor marker, was originally used as a serum marker for colorectal cancer and could reflect the existence of a variety of tumors, such as pancreatic cancer, lung cancer and gastric cancer [10]. Although the three tumor markers are detected preoperatively, they are hardly used for the diagnosis of GC because of the poor sensitivity and specificity $[11,12]$. However, they can be applied in the evaluation of therapeutic effect, monitoring of recurrence and prediction of prognosis [13, 14].

There are amount of studies focused on the relationship between tumor markers and GC, of which only a few studies explored the correlation between preoperative serum tumor markers and clinicopathological traits and survival of GC patients. According to previous studies, there is no doubt that preoperative serum CA19-9 and CEA levels are correlated with TNM stage (based on American Joint Committee on Cancer (AJCC) $7^{\text {th }}$ edition) [15]. As to other clinicopathological traits, including tumor location, tumor size, microscopic type and differentiation grade, there are still no definitive conclusions. In this study, we explored the relationship between preoperative serum tumor markers (CA125, CA19-9 and CEA) and clinicopathological traits and prognostic information of GC patients.

\section{RESULTS}

\section{The baseline of clinicopathological traits in the training and the validation corhorts}

In this study, a total of $1024 \mathrm{GC}$ patients were enrolled in the training cohort, and there were 668 patients in the validation cohort. We first compared the clinicopathological traits and therapy information between the training and the validation cohorts. And the result showed that the main clinical information were similar between the two cohorts, except adjuvant chemotherapy, tumor location, differentiation grade and $\mathrm{M}$ stage (Table 1). The training cohort included more M1 patients and less patients with adjuvant chemotherapy than the validation cohort.

\section{Association between preoperative serum tumor markers and clinicopathological traits}

The relationship between preoperative serum tumor markers and clinicopathological traits in the training cohort was summarized in Table 2 . The positive rates of serum tumor markers were $42.3 \%$ for CA125, $20.0 \%$ for CA19-9 and $19.2 \%$ for CEA. When three tumor markers were combined, the triple-positive rate was only $3.7 \%$ ( $n$ $=38$ ). In univariate analysis, the higher positive rates of CA125, CA19-9 and CEA were significantly associated with larger tumor size, more advanced macroscopic type and pTNM stage (all $P<0.05$ ). In the positive group of each tumor marker, the proportions of stage III-IV were $67.9 \%$ for CA125 (+), $77.6 \%$ for CA19-9 (+) and $77.7 \%$ for CEA $(+)$. When three tumor markers were combined, the proportion of stage III-IV rose to $89.5 \%$. As to lymph node metastasis, the proportions of $\mathrm{N}+$ were $74.8 \%$ for CA125 (+), 84.9\% for CA19-9 (+) and 84.3\% for CEA $(+)$, and it rose to $94.7 \%$ when three tumor markers were simultaneously positive (Table 3). CA125 and CA19-9 had significantly higher positive rate in female patients than that in male patients $(P=0.001$ and $P=0.009$, respectively), while CEA had remarkably higher positive rate in male patients than that in female patients $(P=$ $0.027)$. However, CA19-9 had obviously higher positive rate in older patients than that in younger patients $(P=$ 0.043). Similarly, in the validation cohort, the higher positive rates of all three tumor markers were significantly associated with larger tumor size, more advanced macroscopic type and pTNM stage (all $P<0.05$ )(Table 4).

The multivariate analysis revealed that, in the training cohort, the positive rate of CA19-9 were significantly associated with age $(P=0.008)$. Gender, pT stage and pN stage (all $P<0.05)$ were associated with all three tumor markers. In the validation cohort, gender $(P$ $=0.012)$ and tumor size $(P<0.001)$ were independently related to CA125. pT stage $(P=0.019)$ and $\mathrm{pN}$ stage $(P=0.001)$ were independently associated with CA199. However, gender $(P<0.001)$, macroscopic type $(P$ $=0.022)$ and $\mathrm{pN}$ stage $(P=0.019)$ were independently related to CEA (Table 5).

\section{The prognostic significance of preoperative serum tumor markers in the training cohort}

Nine hundred and twenty-four patients (924/1024, $90.2 \%$ ) were followed up and analyzed in prognosis with median survival time of $85.1(0.3-129.9)$ months. For all 924 patients, the 1-, 2-, 3- and 4-cumulative overall survival rates were $77 \%, 67 \%, 59 \%$ and $55 \%$, respectively. For patients in the positive groups of CA125, CA19-9 and CEA, the 3-year survival rates were $51 \%, 44 \%$ and $43 \%$, respectively, compared with $66 \%, 63 \%$ and $63 \%$ for patients in the negative groups of these markers, while the rate in patients with three markers simultaneously positive was $29 \%$.

Univariate and multivariate analysis for prognostic factors were shown in Table 6. Compared with the positive groups by Kaplan-Meier analysis, the negative groups of all three tumor markers showed significantly higher survival rates, respectively (Figure 1A, 1B, 1C, all $P<$ $0.001)$. In univariate survival analysis, age $(P=0.016)$, 
Table 1: The clinicopathological traits of patients in the training and the validation cohorts

\begin{tabular}{|c|c|c|c|c|}
\hline Patient characteristic & & \begin{tabular}{|l|} 
Training \\
$n(\%)$
\end{tabular} & \begin{tabular}{|l|} 
Validation \\
$n(\%)$
\end{tabular} & $P$ \\
\hline \multirow[t]{2}{*}{ Gender } & Male & $702(68.6)$ & $454(68.0)$ & 0.799 \\
\hline & Female & $322(31.4)$ & $214(32.0)$ & \\
\hline \multirow[t]{2}{*}{ Age (ys) } & $<60$ & $564(55.1)$ & $339(50.7)$ & 0.081 \\
\hline & $\geq 60$ & $460(44.9)$ & $329(49.3)$ & \\
\hline \multirow[t]{3}{*}{ Resection pattern } & Distal & $575(56.2)$ & $368(55.1)$ & 0.132 \\
\hline & Total & $280(27.3)$ & $208(31.1)$ & \\
\hline & Proximal & $169(16.5)$ & $92(13.8)$ & \\
\hline \multirow[t]{2}{*}{ Lymphadenectomy } & D1/D1+ & $550(53.7)$ & $368(55.1)$ & 0.578 \\
\hline & $\mathrm{D} 2 / \mathrm{D} 2+$ & $474(46.3)$ & $300(44.9)$ & \\
\hline \multirow[t]{2}{*}{ Chemotherapy } & No & $649(63.4)$ & $348(52.1)$ & $<0.001$ \\
\hline & Yes & $375(36.6)$ & $320(47.9)$ & \\
\hline \multirow[t]{4}{*}{ Tumor location } & Upper & $252(24.6)$ & $187(28.0)$ & 0.002 \\
\hline & Middle & $223(21.8)$ & $97(14.5)$ & \\
\hline & Lower & $529(51.7)$ & $373(55.8)$ & \\
\hline & Whole & $20(2.0)$ & $11(1.6)$ & \\
\hline \multirow[t]{4}{*}{ Tumor size $(\mathrm{cm})$} & $\leq 2$ & $140(13.7)$ & $81(12.1)$ & 0.106 \\
\hline & $2-5$ & 487 (47.6) & $300(44.9)$ & \\
\hline & $5-8$ & $308(30.1)$ & $227(34.0)$ & \\
\hline & $>8$ & $89(8.7)$ & $60(9.0)$ & \\
\hline \multirow[t]{5}{*}{ Macroscopic type } & Type- 0 & $161(15.7)$ & $90(13.5)$ & 0.050 \\
\hline & Type-1 & $38(3.7)$ & $29(4.3)$ & \\
\hline & Type-2 & $443(43.3)$ & 273 (40.9) & \\
\hline & Type-3 & $333(32.5)$ & $225(33.7)$ & \\
\hline & Type-4 & 49 (4.8) & $51(7.6)$ & \\
\hline \multirow[t]{3}{*}{ Differentiation grade } & Well & $17(1.7)$ & $5(0.7)$ & 0.001 \\
\hline & Moderate & $193(18.8)$ & $91(13.6)$ & \\
\hline & Poor & $814(79.5)$ & $572(85.6)$ & \\
\hline \multirow[t]{4}{*}{ pT stage } & $\mathrm{T} 1$ & $188(18.4)$ & $122(18.3)$ & 0.055 \\
\hline & $\mathrm{T} 2$ & $120(11.7)$ & $87(13.0)$ & \\
\hline & $\mathrm{T} 3$ & $106(10.4)$ & $106(15.9)$ & \\
\hline & $\mathrm{T} 4$ & $610(59.6)$ & $353(52.8)$ & \\
\hline \multirow[t]{5}{*}{ pN stage } & N0 & $310(30.3)$ & $206(30.8)$ & 0.378 \\
\hline & N1 & $199(19.4)$ & 115 (17.2) & \\
\hline & N2 & 191 (18.7) & $114(17.1)$ & \\
\hline & $\mathrm{N} 3 \mathrm{a}$ & $216(21.1)$ & 145 (21.7) & \\
\hline & $\mathrm{N} 3 \mathrm{~b}$ & $108(10.5)$ & $88(13.2)$ & \\
\hline \multirow[t]{2}{*}{ M stage } & M0 & 937 (91.5) & 629 (94.2) & 0.042 \\
\hline & M1 & $87(8.5)$ & $39(5.8)$ & \\
\hline \multirow[t]{4}{*}{ pTNM stage } & I & 221 (21.6) & $141(21.1)$ & 0.240 \\
\hline & II & $201(19.6)$ & $151(22.6)$ & \\
\hline & III & $515(50.3)$ & 337 (50.4) & \\
\hline & IV & $87(8.5)$ & $39(5.8)$ & \\
\hline
\end{tabular}


Table 2: Correlation between preoperative serum tumor makers and major clinicopathological traits in the training cohort

\begin{tabular}{|c|c|c|c|c|c|c|c|c|}
\hline \multicolumn{2}{|l|}{ Patient characteristics } & \multirow{2}{*}{\begin{tabular}{|l|}
$\begin{array}{l}\text { Cases } \\
\boldsymbol{n}(\mathbf{\%})\end{array}$ \\
$702(68.6)$ \\
\end{tabular}} & \multirow{2}{*}{$\begin{array}{l}\text { CA125 (+) } \\
\boldsymbol{n} \mathbf{( \% )} \\
272(38.7)\end{array}$} & \multirow{2}{*}{$\begin{array}{l}P \\
0.001\end{array}$} & \multirow{2}{*}{$\begin{array}{l}\begin{array}{l}\text { CA19-9 (+) } \\
\boldsymbol{n}(\mathbf{\%})\end{array} \\
125(17.8)\end{array}$} & \multirow{2}{*}{\begin{tabular}{|l|}
$P$ \\
0.009
\end{tabular}} & \multirow{2}{*}{\begin{tabular}{|l|}
$\begin{array}{l}\text { CEA (+) } \\
n(\%)\end{array}$ \\
$148(21.1)$ \\
\end{tabular}} & \multirow{2}{*}{\begin{tabular}{|l|}
$P$ \\
0.027 \\
\end{tabular}} \\
\hline Gender & Male & & & & & & & \\
\hline & Female & $322(31.4)$ & $161(50.0)$ & & $80(24.8)$ & & $49(15.2)$ & \\
\hline \multirow[t]{2}{*}{ Age (ys) } & $<60$ & $564(55.1)$ & $233(41.3)$ & 0.485 & $100(17.7)$ & 0.043 & $99(17.6)$ & 0.130 \\
\hline & $\geq 60$ & $460(44.9)$ & $200(43.5)$ & & $105(22.8)$ & & $98(21.3)$ & \\
\hline \multirow[t]{4}{*}{ Tumor location } & Upper & $252(24.6)$ & $93(36.9)$ & 0.029 & $50(19.8)$ & 0.012 & $61(24.2)$ & 0.146 \\
\hline & Middle & $223(21.8)$ & $110(49.3)$ & & $56(25.1)$ & & $40(17.9)$ & \\
\hline & Lower & $529(51.7)$ & $219(41.4)$ & & $91(17.2)$ & & $92(17.4)$ & \\
\hline & Whole & $20(2.0)$ & $11(55.0)$ & & $8(40.0)$ & & $4(20.0)$ & \\
\hline \multirow[t]{4}{*}{ Tumor size $(\mathrm{cm})$} & $\leq 2$ & $140(13.7)$ & $49(35.0)$ & $<0.001$ & $14(10.0)$ & $<0.001$ & $13(9.3)$ & $<0.001$ \\
\hline & $2-5$ & 487 (47.6) & $180(37.0)$ & & $90(18.5)$ & & $83(17.0)$ & \\
\hline & $5-8$ & $308(30.1)$ & $162(52.6)$ & & $76(24.7)$ & & $78(25.3)$ & \\
\hline & $>8$ & $89(8.7)$ & $42(47.2)$ & & $25(28.1)$ & & $23(25.8)$ & \\
\hline \multirow[t]{5}{*}{ Macroscopic type } & Type- 0 & $161(15.7)$ & $53(32.9)$ & 0.002 & $19(11.8)$ & 0.001 & $15(9.3)$ & 0.012 \\
\hline & Type-1 & $38(3.7)$ & $14(36.8)$ & & $7(18.4)$ & & $9(23.7)$ & \\
\hline & Type-2 & $443(43.3)$ & $185(41.8)$ & & 87 (19.6) & & $90(20.3)$ & \\
\hline & Type-3 & $333(32.5)$ & $159(47.7)$ & & $76(22.8)$ & & $73(21.9)$ & \\
\hline & Type-4 & 49 (4.8) & $22(44.9)$ & & $16(32.7)$ & & $10(20.4)$ & \\
\hline \multirow[t]{3}{*}{ Differentiation grade } & Well & $17(1.7)$ & $3(17.6)$ & 0.016 & $0(0.0)$ & 0.064 & $2(11.8)$ & 0.413 \\
\hline & Moderate & $193(18.8)$ & $71(36.8)$ & & $33(17.1)$ & & $43(22.3)$ & \\
\hline & Poor & $814(79.5)$ & $359(44.1)$ & & $172(21.1)$ & & $152(18.7)$ & \\
\hline \multirow[t]{4}{*}{ pT stage } & $\mathrm{T} 1$ & $188(18.4)$ & $60(31.9)$ & $<0.001$ & $19(10.1)$ & $<0.001$ & $14(7.4)$ & $<0.001$ \\
\hline & $\mathrm{T} 2$ & $120(11.7)$ & $40(33.3)$ & & $16(12.3)$ & & $19(15.8)$ & \\
\hline & T3 & $106(10.4)$ & $47(44.3)$ & & $13(12.3)$ & & $15(14.2)$ & \\
\hline & T4 & $610(59.6)$ & $286(46.9)$ & & $157(25.7)$ & & $149(24.4)$ & \\
\hline \multirow[t]{5}{*}{ pN stage } & N0 & $310(30.3)$ & $109(35.2)$ & $<0.001$ & $31(10.0)$ & $<0.001$ & $31(10.0)$ & $<0.001$ \\
\hline & N1 & $199(19.4)$ & $73(36.7)$ & & $39(19.6)$ & & $33(16.6)$ & \\
\hline & N2 & $191(18.7)$ & $78(40.8)$ & & $39(20.4)$ & & $42(22.0)$ & \\
\hline & N3a & $216(21.1)$ & $109(50.5)$ & & $55(25.5)$ & & $60(27.8)$ & \\
\hline & $\mathrm{N} 3 \mathrm{~b}$ & $108(10.5)$ & $64(59.3)$ & & $41(38.0)$ & & $31(28.7)$ & \\
\hline \multirow[t]{2}{*}{ M stage } & M0 & $937(91.5)$ & $381(40.7)$ & 0.001 & $173(18.5)$ & $<0.001$ & $167(17.8)$ & $<0.001$ \\
\hline & M1 & $87(8.5)$ & $52(59.8)$ & & $32(36.8)$ & & $30(34.5)$ & \\
\hline \multirow[t]{4}{*}{ pTNM stage } & $\mathrm{I}$ & $221(21.6)$ & $69(31.2)$ & $<0.001$ & $22(10.0)$ & $<0.001$ & $19(8.6)$ & $<0.001$ \\
\hline & II & $201(19.6)$ & $70(34.8)$ & & $24(11.9)$ & & $25(12.4)$ & \\
\hline & III & $515(50.3)$ & $242(47.0)$ & & $127(24.7)$ & & $123(23.9)$ & \\
\hline & IV & $87(8.5)$ & $52(59.8)$ & & $32(36.8)$ & & $30(34.5)$ & \\
\hline
\end{tabular}


Table 3: Correlation between tumor marker positivity and lymph node metastasis and pTNM stage in the training cohort

\begin{tabular}{|l|l|l|l|l|}
\hline & Triple-positive & CA125 $(+)$ & CA19-9 $(+)$ & CEA (+) \\
\hline N0 & $2(5.3)$ & $109(25.2)$ & $31(15.1)$ & $31(15.7)$ \\
\hline $\mathrm{N}+$ & $36(94.7)$ & $324(74.8)$ & $174(84.9)$ & $166(84.3)$ \\
\hline P & & $\mathbf{0 . 0 0 6}$ & 0.103 & 0.089 \\
\hline I-II & $4(10.5)$ & $139(32.1)$ & $46(22.4)$ & $44(22.3)$ \\
\hline III-IV & $34(89.5)$ & $294(67.9)$ & $159(77.6)$ & $153(77.7)$ \\
\hline P & & $\mathbf{0 . 0 0 6}$ & 0.095 & 0.098 \\
\hline
\end{tabular}

tumor location $(P<0.001)$, tumor size $(P<0.001)$, macroscopic type $(P<0.001)$, differentiation grade $(P$ $=0.021), \mathrm{pT}$ stage $(P<0.001), \mathrm{pN}$ stage $(P<0.001)$, M stage $(P<0.001)$ and pTNM stage $(P<0.001)$ were significantly associated with prognosis. In a multivariate analysis, age $(P=0.011)$, tumor size $(P<0.001)$, pTNM stage $(P<0.001)$ and CA $125(P=0.026)$ were independent prognostic factors.

A

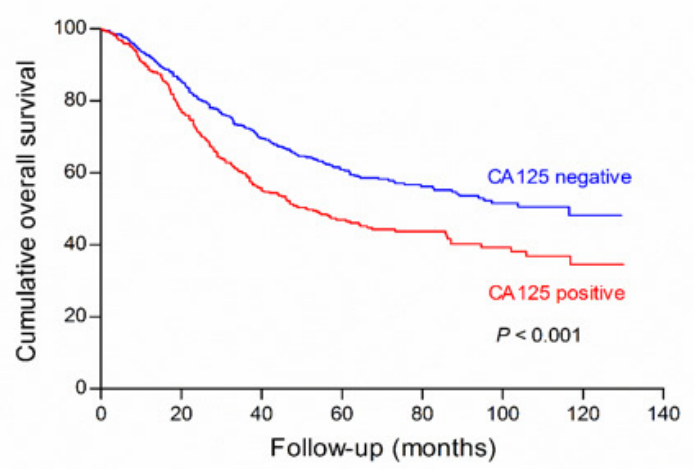

C

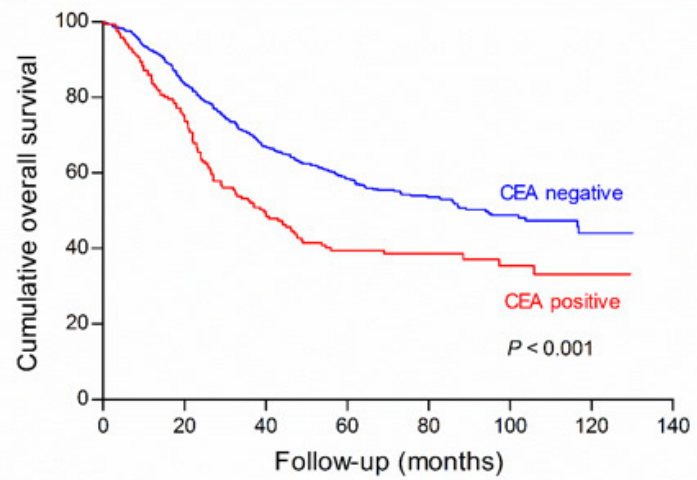

The prognostic value of nomogram with preoperative serum tumor markers and clinicopathological traits in the training cohort

We made a further analysis by using nomogram to predict 3-year overall survival rate of individual patient. When one preoperative serum tumor marker positivity was

Figure 1: Survival analysis of subgroups of CA125 (A), CA19-9 (B), CEA (C) and their combined detection (D) in the training cohort. There were significant differences on survival outcomes between positive and negative subgroups in CA125, CA19-9, CEA and their combined detection, respectively (all $P<0.001$ )

B

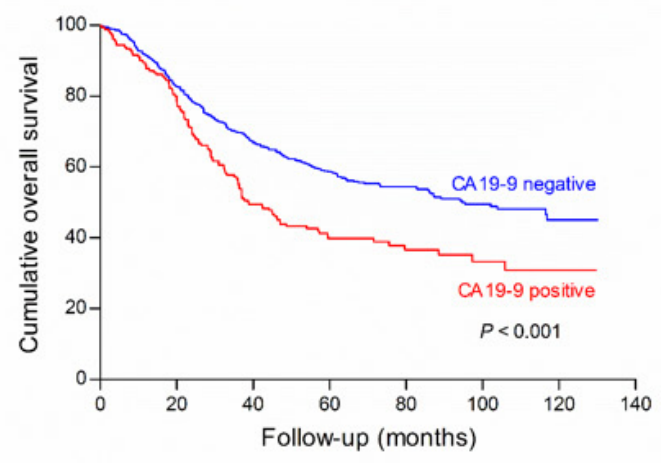

D

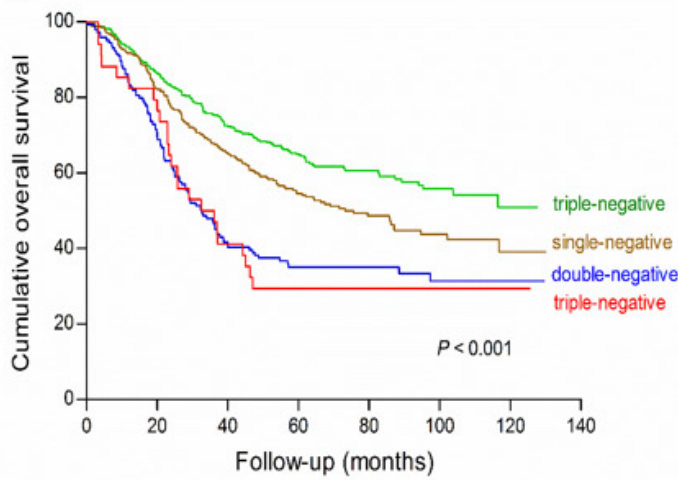


Table 4: Correlation between preoperative serum tumor makers and major clinicopathological traits in the validation cohort

\begin{tabular}{|c|c|c|c|c|c|c|c|c|}
\hline Patient characteristic & & \begin{tabular}{|l|} 
Cases \\
$n(\%)$ \\
\end{tabular} & \begin{tabular}{|l|} 
CA125 (+) \\
$n(\%)$ \\
\end{tabular} & $P$ & \begin{tabular}{|l|} 
CA19-9 (+) \\
$n(\%)$ \\
\end{tabular} & $P$ & \begin{tabular}{|l|l|} 
CEA (+) \\
$n(\%)$
\end{tabular} & $P$ \\
\hline \multirow[t]{2}{*}{ Gender } & Male & $454(68.0)$ & $171(37.7)$ & 0.007 & $97(21.4)$ & 0.278 & $109(24.0)$ & $<0.001$ \\
\hline & Female & $214(32.0)$ & $104(48.6)$ & & $38(17.8)$ & & 24 (11.2) & \\
\hline \multirow[t]{2}{*}{ Age (ys) } & $<60$ & $339(50.7)$ & $142(41.9)$ & 0.701 & $69(20.4)$ & 0.925 & $58(17.1)$ & 0.066 \\
\hline & $\geq 60$ & $329(49.3)$ & $133(40.4)$ & & $66(20.1)$ & & $75(22.8)$ & \\
\hline \multirow[t]{4}{*}{ Tumor location } & Upper & $187(28.0)$ & 78 (41.7) & 0.374 & $46(24.6)$ & 0.293 & $46(24.6)$ & 0.122 \\
\hline & Middle & $97(14.5)$ & $47(48.5)$ & & $20(20.6)$ & & $21(21.6)$ & \\
\hline & Lower & $373(55.8)$ & $145(38.9)$ & & 68 (18.2) & & $63(16.9)$ & \\
\hline & Whole & $11(1.6)$ & $5(45.5)$ & & $1(9.1)$ & & $3(27.3)$ & \\
\hline \multirow[t]{4}{*}{ Tumor size $(\mathrm{cm})$} & $\leq 2$ & $81(12.1)$ & $24(29.6)$ & $<0.001$ & $7(8.6)$ & 0.001 & $12(14.8)$ & 0.004 \\
\hline & $2-5$ & $300(44.9)$ & $101(33.7)$ & & $56(18.7)$ & & 47 (15.7) & \\
\hline & $5-8$ & $227(34.0)$ & $117(51.5)$ & & $56(24.7)$ & & $61(26.9)$ & \\
\hline & $>8$ & $60(9.0)$ & $33(55.0)$ & & $16(26.7)$ & & $13(21.7)$ & \\
\hline \multirow[t]{5}{*}{ Macroscopic type } & Type- 0 & $90(13.5)$ & $21(23.3)$ & $<\mathbf{0 . 0 0 1}$ & $7(7.8)$ & 0.002 & $8(8.9)$ & 0.003 \\
\hline & Type-1 & $29(4.3)$ & $16(55.2)$ & & $4(13.8)$ & & $4(13.8)$ & \\
\hline & Type-2 & $273(40.9)$ & $108(39.6)$ & & $56(20.5)$ & & $56(20.5)$ & \\
\hline & Type-3 & $225(33.7)$ & $98(35.6)$ & & $57(25.3)$ & & 49 (21.8) & \\
\hline & Type-4 & $51(7.6)$ & $32(62.7)$ & & $11(21.6)$ & & $16(31.4)$ & \\
\hline \multirow[t]{3}{*}{ Differentiation grade } & Well & $5(0.7)$ & $3(60.0)$ & 0.452 & $0(0.0)$ & 0.131 & $0(0.0)$ & 0.944 \\
\hline & Moderate & $91(13.6)$ & $33(36.3)$ & & $14(15.4)$ & & $19(20.9)$ & \\
\hline & Poor & $572(85.6)$ & $239(41.8)$ & & $121(21.2)$ & & $114(19.9)$ & \\
\hline \multirow[t]{4}{*}{ pT stage } & $\mathrm{T} 1$ & $122(18.3)$ & 35 (28.7) & $<0.001$ & $12(9.8)$ & $<0.001$ & 14 (11.5) & 0.017 \\
\hline & $\mathrm{T} 2$ & $87(13.0)$ & $30(34.5)$ & & $14(16.1)$ & & $19(21.8)$ & \\
\hline & $\mathrm{T} 3$ & $106(15.9)$ & $41(38.7)$ & & $14(13.2)$ & & $19(17.9)$ & \\
\hline & $\mathrm{T} 4$ & $353(52.8)$ & $169(47.9)$ & & $95(26.9)$ & & $81(22.9)$ & \\
\hline \multirow[t]{5}{*}{ pN stage } & N0 & $206(30.8)$ & $59(28.6)$ & 0.001 & $15(7.3)$ & $<0.001$ & $23(11.2)$ & 0.001 \\
\hline & N1 & $115(17.2)$ & $56(48.7)$ & & $29(25.2)$ & & $29(25.2)$ & \\
\hline & $\mathrm{N} 2$ & $114(17.1)$ & $55(48.2)$ & & $24(21.1)$ & & $24(21.1)$ & \\
\hline & $\mathrm{N} 3 \mathrm{a}$ & $145(21.7)$ & $61(42.1)$ & & $42(29.0)$ & & $33(22.8)$ & \\
\hline & $\mathrm{N} 3 \mathrm{~b}$ & $88(13.2)$ & $44(50.0)$ & & $25(28.4)$ & & $24(27.3)$ & \\
\hline \multirow[t]{2}{*}{ M stage } & M0 & $629(94.2)$ & $252(40.1)$ & 0.020 & $126(20.0)$ & 0.646 & $119(18.9)$ & 0.010 \\
\hline & M1 & $39(5.8)$ & $23(59.0)$ & & $9(23.1)$ & & $14(35.9)$ & \\
\hline \multirow[t]{4}{*}{ pTNM stage } & I & $141(21.1)$ & 39 (27.7) & $<0.001$ & $12(8.5)$ & $<0.001$ & $19(13.5)$ & 0.005 \\
\hline & II & $151(22.6)$ & $54(35.8)$ & & 19 (12.6) & & 28 (18.5) & \\
\hline & III & $337(50.4)$ & $159(47.2)$ & & $95(28.2)$ & & $72(21.4)$ & \\
\hline & IV & $39(5.8)$ & $23(59.0)$ & & $9(23.1)$ & & $14(35.9)$ & \\
\hline
\end{tabular}


Table 5: Multivariate analysis of preoperative serum tumor makers with clinicopathological traits in the training and the validation cohorts

\begin{tabular}{|c|c|c|c|c|c|c|}
\hline & \multicolumn{2}{|l|}{ CA125 (+) } & \multicolumn{2}{|l|}{ CA19-9 (+) } & \multicolumn{2}{|l|}{ CEA $(+)$} \\
\hline & OR $(95 \% \mathrm{CI})$ & $P$ & OR $(95 \% \mathrm{CI})$ & $\boldsymbol{P}$ & OR $(95 \% \mathrm{CI})$ & $P$ \\
\hline \multicolumn{7}{|l|}{ Training cohort } \\
\hline Gender & $1.614(1.231-2.115)$ & 0.001 & $1.650(1.184-2.299)$ & 0.003 & $0.662(0.461-0.951)$ & 0.026 \\
\hline Age & - & - & $1.550(1.124-2.139)$ & 0.008 & - & - \\
\hline pT stage & $1.138(1.005-1.289)$ & 0.041 & $1.264(1.061-1.507)$ & 0.009 & $1.315(1.099-1.572)$ & 0.003 \\
\hline pN stage & $1.197(1.077-1.331)$ & 0.001 & $1.345(1.180-1.532)$ & $<0.001$ & $1.277(1.120-1.456)$ & $<0.001$ \\
\hline \multicolumn{7}{|l|}{ Validation cohort } \\
\hline Gender & $1.538(1.101-2.148)$ & 0.012 & - & & $0.372(0.229-0.603)$ & $<0.001$ \\
\hline Tumor size & $1.596(1.310-1.945)$ & $<0.001$ & - & & - & \\
\hline Macroscopic type & - & & - & & $1.279(1.036-1.580)$ & 0.022 \\
\hline pT stage & - & & $1.289(1.043-1.592)$ & 0.019 & - & \\
\hline pN stage & - & & $1.288(1.106-1.501)$ & 0.001 & $1.194(1.029-1.385)$ & 0.019 \\
\hline
\end{tabular}

$\mathrm{OR}=$ odds ratio; $\mathrm{CI}=$ confidence interval.

considered as a single prognostic factor, the number of positive serum tumor markers (NPTM) was likely to be a parameter in nomogram. By using Cox regression test, age $(P=0.019)$, tumor size $(P<0.001)$, pTNM stage $(P<$ $0.001)$ and NPTM $(P=0.021, \mathrm{HR}=1.292,95 \%$ CI 1.040
- 1.604) were included in the nomogram (Figure 2). The nomogram indicated that age $\geq 60$, large NPTM ( $n=2$ or 3 ), large tumor size and advanced pTNM stage were the poor prognostic factors, but pTNM stage was still a most powerful one. The result of the nomogram was analogous

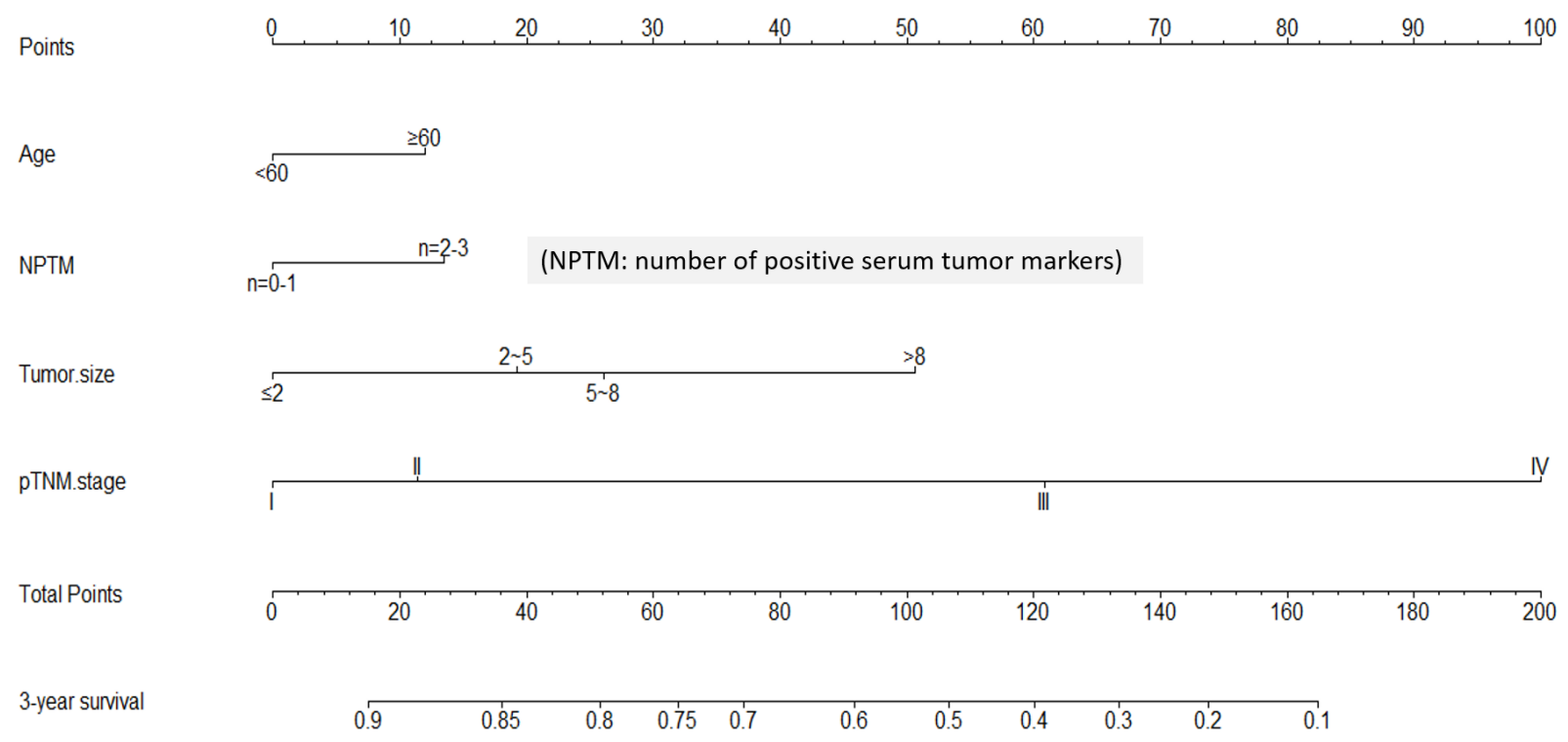

Figure 2: Nomogram of NPTM and clinicopathological traits in the training cohort 
Table 6: Univariate and multivariate analysis of prognostic risk factors for overall survival in the training and the validation cohorts

\begin{tabular}{|c|c|c|c|c|c|c|}
\hline \multirow{3}{*}{ Risk factors } & \multicolumn{3}{|c|}{ Training cohort $(n=1024)$} & \multicolumn{3}{|c|}{ Validation cohort $(n=668)$} \\
\hline & \multirow{2}{*}{\begin{tabular}{|l} 
Univariate \\
$P$ value
\end{tabular}} & \multicolumn{2}{|l|}{ Multivariate } & \multirow{2}{*}{\begin{tabular}{|l} 
Univariate \\
$P$ value
\end{tabular}} & \multicolumn{2}{|l|}{ Multivariate } \\
\hline & & HR $(95 \% C I)$ & $P$ value & & HR $(95 \% C I)$ & $P$ value \\
\hline Gender & 0.401 & - & - & 0.462 & - & - \\
\hline Age (ys) & 0.016 & $1.273(1.057-1.533)$ & 0.011 & 0.590 & - & - \\
\hline Tumor location & $<0.001$ & - & & 0.001 & - & - \\
\hline Tumor size $(\mathrm{cm})$ & $<0.001$ & $1.298(1.142-1.476)$ & $<0.001$ & $<0.001$ & $1.366(1.100-1.697)$ & 0.005 \\
\hline Macroscopic type & $<0.001$ & - & & $<0.001$ & $1.280(1.054-1.554)$ & 0.013 \\
\hline Differentiation grade & 0.021 & - & & 0.022 & - & - \\
\hline pTNM stage & $<0.001$ & $2.183(1.900-2.508)$ & $<0.001$ & $<0.001$ & $2.101(1.655-2.668)$ & $<0.001$ \\
\hline CA125 & $<0.001$ & $1.238(1.026-1.492)$ & 0.026 & $<0.001$ & $1.519(1.132-2.039)$ & 0.005 \\
\hline CA19-9 & $<0.001$ & - & & $<0.001$ & $1.431(1.051-1.949)$ & 0.023 \\
\hline CEA & $<0.001$ & - & & 0.001 & - & - \\
\hline NPTM & $<0.001$ & $1.292(1.040-1.604)$ & 0.021 & $<0.001$ & $1.502(1.099-2.052)$ & 0.011 \\
\hline
\end{tabular}

$\mathrm{HR}=$ hazard ratio; $\mathrm{CI}=$ confidence interval; NPTM $=$ number of positive serum tumor markers.

to those of above-mentioned multivariate analysis. The calibration curve of nomogram showed that the predictive probability of 3-year survival was closely to the actual 3-year survival (Figure 3).

Subsequently, we compared the predictive accuracy of prognosis between the nomogram and TNM staging system (only pTNM stage). The C-index of nomogram was 0.718 , compared with 0.689 of TNM staging system. The difference between nomogram and TNM staging system was significant $(P<0.001)$.

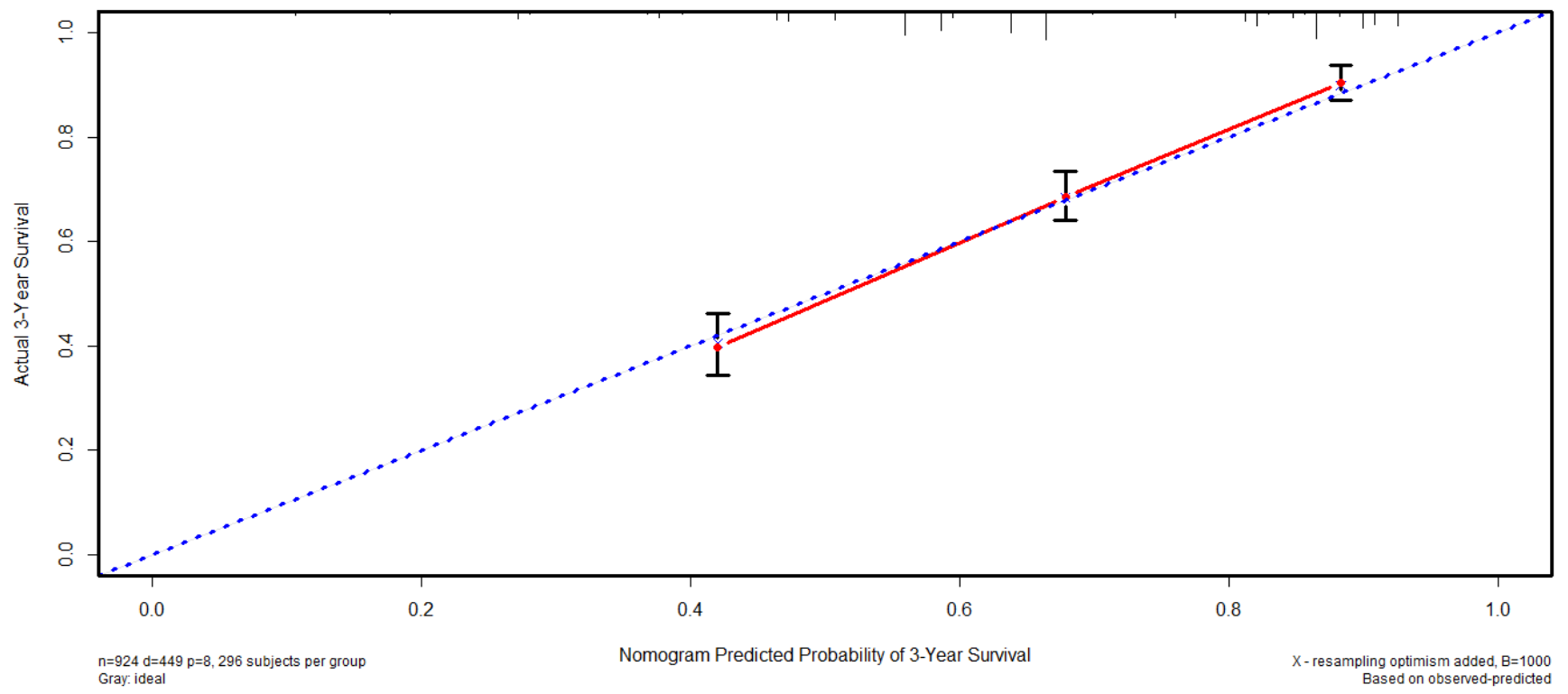

Figure 3: Calibration curve of nomogram in the training cohort 


\section{Validation of preoperative serum tumor markers in an independent cohort}

Six hundred and fifty patients $(650 / 668,97.3 \%)$ were followed up with median survival time of 30.9 (0.5$47.0)$ months in the validation cohort. For all 650 patients, the 1- and 2- cumulative overall survival rates were $78 \%$ and $69 \%$, respectively. The negative groups of all three tumor markers had significantly higher survival rates than the positive groups, respectively (Figure 4A, 4B, $4 \mathrm{C}$, all $P<0.001$ ). In univariate survival analysis, tumor location, tumor size, macroscopic type, differentiation grade, $\mathrm{pT}$ stage, $\mathrm{pN}$ stage, $\mathrm{M}$ stage and $\mathrm{pTNM}$ stage were significantly associated with prognosis (all $P<0.05$ ). In a multivariate analysis, tumor size $(P=0.005)$, macroscopic type $(P=0.013)$, pTNM stage $(P<0.001)$, CA $125(P$ $=0.005)$ and CA 19-9 $(P=0.023)$ were independent prognostic factors.

By using Cox regression test, macroscopic type $(P=0.013)$, tumor size $(P=0.003)$, pTNM stage $(P<$ $0.001)$ and NPTM $(P=0.011, \mathrm{HR}=1.3502,95 \%$ CI 1.099 - 2.052) were included in the nomogram (Figure 5). The result of the nomogram was analogous to those of above- mentioned multivariate analysis. The calibration curve of nomogram showed that the predictive probability of 2-year survival was closely to the actual 2-year survival (Figure 6). The C-index of nomogram was 0.747 , compared with 0.694 of TNM staging system. The difference between nomogram and TNM staging system was significant $(P$ $<0.001)$.

\section{DISCUSSION}

Serum tumor markers are quite commonly detected before and after surgery for most kinds of tumors. Finding a specific tumor marker will help to increase the accuracy of diagnosis and predict the prognosis of $\mathrm{GC}$ patients, however, the role of existing tumor markers in $\mathrm{GC}$ is still controversial. In addition to some routinely applied tumor markers, such as $\alpha$-Fetoprotein (AFP), CEA, CA19-9 and carbohydrate antigen 72-4 (CA72-4), there are still many other tumor markers used in different hospitals [16]. According to the previous studies, those serum tumor markers showed poor sensitivity and specificity of diagnosis. Nevertheless, the prognostic value of serum tumor markers was supported by many previous studies.
A

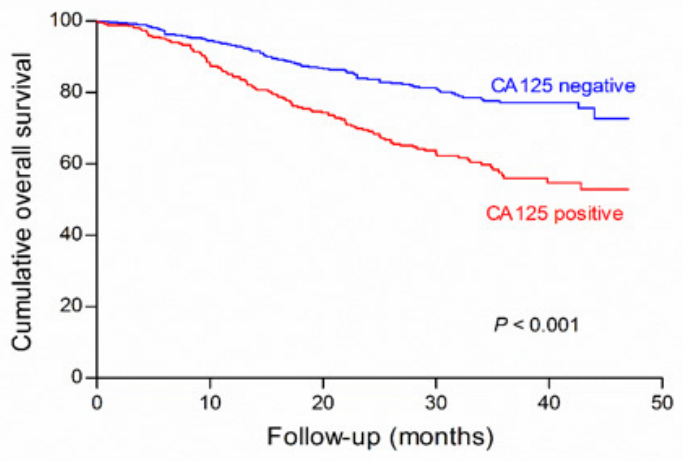

C

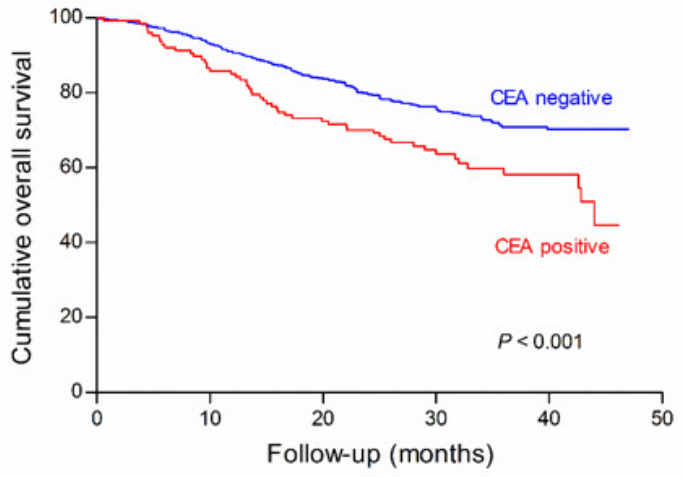

B

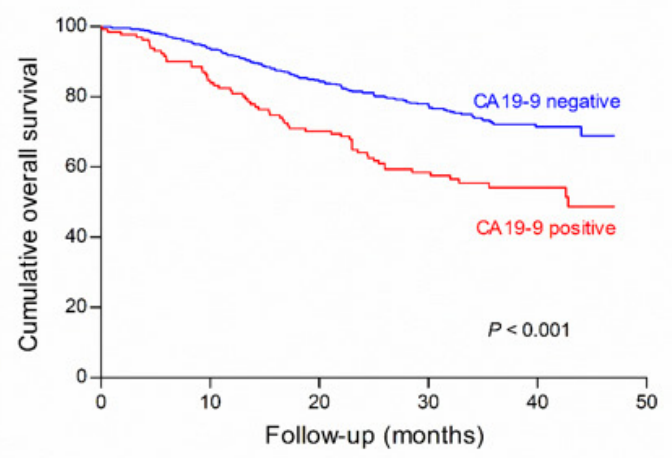

D

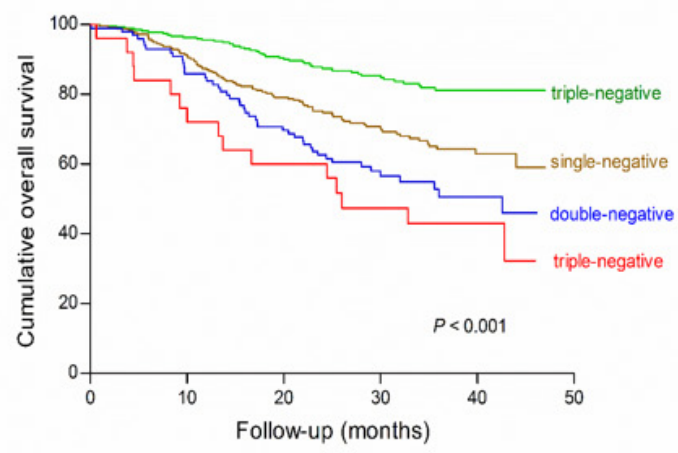

Figure 4: Survival analysis of subgroups of CA125 (A), CA19-9 (B), CEA (C) and their combined detection (D) in the validation cohort. There were significant differences on survival outcomes between positive and negative subgroups in CA125, CA19-9, CEA and their combined detection, respectively (all $P<0.001$ ) 
All enrolled patients in our study were pathologically diagnosed as GC, and our purpose was to explore the prognostic value of preoperative serum tumor markers in GC patients, therefore, the cut-off values of three tumor markers were recalculated. The cut-off values of tumor markers in previous studies were defined by the manufacturer recommendation or calculated by the receiver operating characteristic curve. X-tile plot was applied in our study as it is a time-dependent cut-off value analysis based on the survival information. The new cutoff values of CA125, CA19-9 and CEA were 13.72 U/ $\mathrm{ml}, 23.36 \mathrm{U} / \mathrm{ml}$ and $4.28 \mathrm{ng} / \mathrm{ml}$, respectively. The positive rate of CA19-9 was reported from $17.1 \%$ to $55.8 \%$, and CEA from $16.1 \%$ to $46.6 \%[15,17-21]$, and the positive rates of these two markers in this study were within these ranges (CA19-9: 20.0\%, CEA: 19.2\%). The positive rate of CA1 25 was $42.3 \%$, which was much higher than other studies with the range between $21 \%$ and $31.1 \%$. This big difference might due to that the recalculated cut-off value of CA125 in our study was obviously lower than that of other studies (20 - $35 \mathrm{U} / \mathrm{ml})$ [12, 22, 23].

In a previous study by Erdal et al, which enrolled 106 GC patients, the result showed that there was no significant correlation between $\mathrm{pT}$ stage and $\mathrm{pN}$ stage and serum CA125, CA 19-9 and CEA levels. Moreover, only elevated CEA was significantly associated with $M$ stage [24]. We also analyzed the association between preoperative serum tumor markers and clinicopathological traits, and found that $\mathrm{pT}$ stage, $\mathrm{pN}$ stage, $\mathrm{M}$ stage and pTNM stage were significantly correlated with the positivity of three tumor markers, which was consistent with previous studies $[17,25]$. Our result indicated that GC patients with positive tumor markers might suffer from more advanced pathological stage. When three tumor markers were combined, the proportion of stage III-IV and lymph node metastasis in triple-positive group was higher than the proportion in the positive group of each single tumor marker, which was similar to the previous report [26]. The result indicated that combined detection of serum tumor markers could help clinician to make a more accurate preoperative staging. The tumor size larger than $5 \mathrm{~cm}$ might reflect in high possibility of tumor marker positivity (all $P<0.001$ ), which resembled the previous study [27]. The similar result appeared in macroscopic type that positive tumor markers might indicate more advanced macroscopic type.

The three tumor markers exhibited difference in gender, age, tumor location and differentiation grade. The positive CA125 and CA19-9 were more likely to be seen in female patients, while positive CEA was more trend to be seen in male patients. The positive rates of CA125 and CA19-9 were higher in tumors occupying whole stomach than in tumors occupying limited one part of stomach. Our result was in agreement with previous studies that the sites of GC would affect serum marker levels [22, 28], and the reason might be that tumors occupying whole stomach were generally correlated with large tumor size and advanced pathological stage. The positive rates of tumor markers among the GC patients with different differentiation grade were not statistically significant in some previous studies [22, 29]. However, Seok et al reported that positive CEA were related to the

Points
NPTM
Macroscopic.type
Tumor.size
pTNM.stage
Total Points

Figure 5: Nomogram of NPTM and clinicopathological traits in the validation cohort 
grade of differentiation [30], and the present study found that positive CA125 was significantly correlated with differentiation grade. In summary, the differentiation grade has little impact on GC marker levels. In a multivariate analysis by logistic regression, we confirmed that $\mathrm{pT}$ stage, $\mathrm{pN}$ stage and gender were independent correlated factors of positive CA125 and CEA, while pT stage, $\mathrm{pN}$ stage and age were independent correlated factors of positive CA19-9.

In our study, patients in the positive groups of CA125, CA19-9 or CEA had significantly poorer overall survival than patients with negative marker level, which was similar to the previous researches [17, 19]. When three tumor markers were combined, the increasing number of positive tumor markers might indicate a worse overall survival (Figure 1D and 4D). The possible reason might be that tumor markers as prognostic factors showed superimposed effect. In addition to tumor markers, our result showed that clinicopathological traits, including age, tumor location, tumor size, macroscopic type, differentiation grade and pTNM stage, were significantly associated with overall survival. Multivariate analysis showed that tumor size, pTNM stage and CA125 were independent prognostic factors of GC patients. Zhou et al reported that CA125 was an independent prognostic factor of GC patients, which was analogous to our study [31]. However, Liu et al and Tocchi et al found that both CA19-9 and CEA provided independent predictive value in gastric cancer patients $[19,32]$, and Chen et al found that only CEA was an independent prognostic factor. Moreover, some studies even considered that tumor marker could not be an independent prognostic factor [33].
What cause the difference between our results and these studies might be discrepancies in the number of patients, the heterogeneity of enrolled patients, the detection technique and cut-off values.

Nomogram is an intuitionistic and widely applied method to predict the prognosis of individual patient on the basis of some valuable parameters. In our study, we figured out that the nomogram visually showed the impact of some clinicopathological traits on the prognosis of GC patients in both training and validation cohorts. In the light of nomogram, the prognosis of individual patient could be well predicted. In order to maximize the use of serum tumor markers, NPTM was proposed and included in nomogram via a stepwise algorithm. The predictive accuracy of nomogram was well illustrated through calibration curve. Moreover, we compared the predictive accuracy between nomogram and TNM staging system, and the result showed that nomogram with NPTM and other parameters was better than pTNM stage alone. According to the nomogram, however, we still regarded pTNM stage as one of the most important parameter in GC, but more importantly, other indexes like NPTM and tumor size should also be noticed.

There were some limitations to our study. Firstly, this is a retrospective analysis comes from a single center in western China, the results of this study may not represent overall Chinese population well. Secondly, our study did not take in the control groups including health person and patients with benign lesions, and the comparison of serum tumor marker levels in different groups could not be made. Thirdly, CA72-4 and CA15-3 were not routinely tested for GC patients in our hospital,

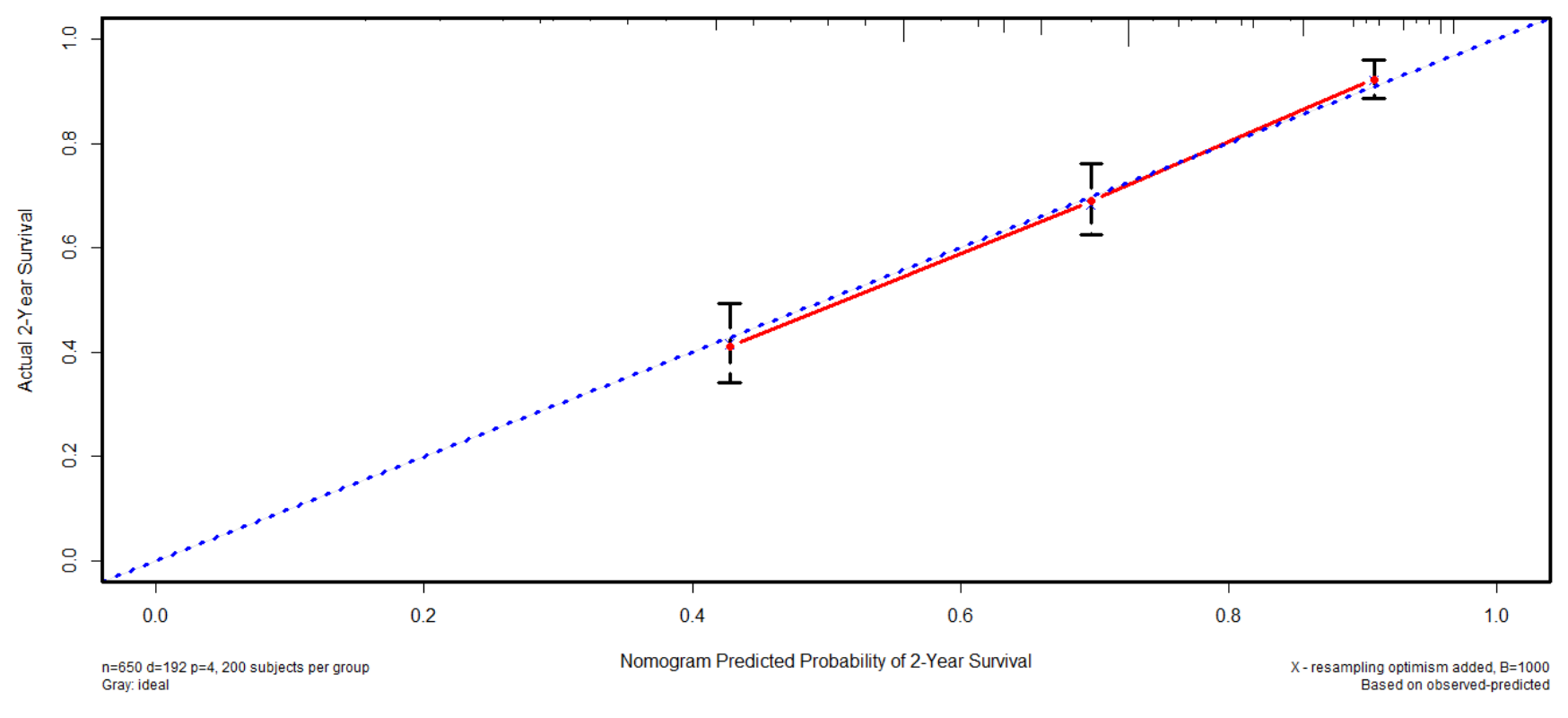

Figure 6: Calibration curve of nomogram in the validation cohort 
therefore they were not analyzed and discussed in this study.

In conclusion, preoperative levels of serum CA125, CA19-9 and CEA were correlated with most of clinicopathological traits, especially pTNM stage. The positivity of CA125, CA19-9 and CEA could provide important prognostic information in GC patients and indicated less favorable outcomes. In addition, CA125 was an independent prognostic factor for GC patients and could be further investigated. Nomogram based on NPTM could accurately predict the prognosis of GC patients.

\section{MATERIALS AND METHODS}

The West China Hospital research ethics committee approved retrospective analysis of anonymous data. Signed patient informed consent was waived per the committee approval, because it was a retrospective analysis.

\section{Patients}

We retrospectively enrolled patients who were diagnosed with resectable primary GC and underwent gastrectomy from January 2005 to December 2013 in the Department of Gastrointestinal Surgery, West China Hospital, Sichuan University. Patients from January 2005 to December 2011 were enrolled into the training cohort, and patients form January 2012 to December 2013 were enrolled into the validation cohort. Patients with neoadjuvant chemotherapy and incomplete clinical data were excluded from the study.

Clinicopathological traits including tumor location, tumor size, microscopic type, differentiation grade, pathological $\mathrm{T}$ stage (pT stage), pathological $\mathrm{N}$ stage (pN stage), M stage and pathological TNM stage (pTNM stage) were collected according to the AJCC $7^{\text {th }}$ edition. In order to analyze the correlation between serum tumor markers and $\mathrm{pN}$ stage, $\mathrm{N}_{+}$stood for the set of N1, N2 and N3.

All enrolled patients received regular follow-up. Outpatient visit was considered to be the optimal choice, meanwhile, telephones and mails were adopted as two main supplementary follow-up methods. According to the National Comprehensive Cancer Network (NCCN) gastric cancer guideline, follow-up was carried out every 3 to 6 months for 1 to 2 years, every 6 to 12 months for next 3 to 5 years, and annually thereafter. The follow-up time of patients in both training and validation cohorts was up to January 2016. The loss of follow-up was mainly due to the change of phone number or home address.

\section{Cut-off value}

The three serum tumor markers were measured within one week before surgery at the clinical laboratory of West China Hospital through Elecsys Modular E170 (Roche Diagnostics, Switzerland). The clinical reference cut-off values of CA125, CA19-9 and CEA were $35 \mathrm{U} / \mathrm{ml}$, $22 \mathrm{U} / \mathrm{ml}$ and $3.4 \mathrm{ng} / \mathrm{ml}$, respectively. Since there was no healthy person (defined as person without GC and other malignancies) as control in this study, we recalculated the cut-off values through X-tile plot, and the recalculated cut-off values of CA125, CA19-9 and CEA were 13.72 $\mathrm{U} / \mathrm{ml}, 23.36 \mathrm{U} / \mathrm{ml}$ and $4.28 \mathrm{ng} / \mathrm{ml}$, respectively. Patients were divided into positive $(+)$ group and negative $(-)$ group for each marker according to the recalculated cutoff values. Patients with tumor marker levels lower than cut-off values were distributed into negative group, and other patients with tumor marker levels higher than cutoff values were distributed into positive group. Using combined detection, patients were divided into four groups: negative group, single-positive group, doublepositive group and triple-positive group.

\section{Statistics}

The statistical analysis was performed using SPSS 22.0 statistical software (SPSS Inc., Chicago, IL, USA). The X-tile plot (Version 3.6.1, Yale University) was used to calculate the cut-off values of serum tumor markers. The $\chi^{2}$, rank sum and Student's T tests were used to evaluate the association between tumor markers and clinicopathological traits for univariate analysis and logistic regression test for multivariate analysis. Kaplan-Meier method and Cox regression test were used to analyze univariate and multivariate prognostic factors, respectively. Nomogram was used to analyze the prognostic value of tumor markers and clinicopathological traits through R for Windows (Version 3.2.0, R Foundation for Statistical Computing). Two-tailed $P$ value less than 0.05 was considered as statistically significance.

\section{ACKNOWLEDGMENTS}

The authors thank the substantial work of Volunteer Team of Gastric Cancer Surgery (VOLTGA), West China Hospital, Sichuan University, China for the assistance in collecting the data.

\section{CONFLICTS OF INTEREST}

The authors indicate no potential conflicts of interest. 


\section{GRANT SUPPORT}

This work was supported by grants from National Natural Science Foundation of China (No. 81372344), Sichuan Province Youth Science \& Technology Innovative Research Team (No.2015TD0009) and West China Hospital 1·3.5 Project.

\section{REFERENCES}

1. Sun XD, Mu R, Zhou YS, Dai XD, Zhang SW, Huangfu XM, Sun J, Li LD, Lu FZ, Qiao YL. Analysis of mortality rate of stomach cancer and its trend in twenty years in China [Article in Chinese]. Zhonghua Zhong Liu Za Zhi. 2004; 26: 4-9.

2. Whiting J, Sano T, Saka M, Fukagawa T, Katai H, Sasako M. Follow-up of GC: a review. Gastric Cancer. 2006; 9: 74-81.

3. Jemal A, Bray F, Center MM, Ferlay J, Ward E, Forman D. Global cancer statistics, CA Cancer J Clin. 2011; 61: 69-90.

4. Duffy MJ, Sturgeon CM, Sölétormos G, Barak V, Molina R, Hayes DF, Diamandis EP, Bossuyt PM. Validation of new cancer biomarkers: a position statement from the European group on tumor markers. Clin Chem. 2015; 61: 809-820.

5. Beketic-Oreskovic L, Maric P, Ozretic P, Oreskovic D, Ajdukovic M, Levanat S. Assessing the clinical significance of tumor markers in common neoplasms. Front Biosci (Elite Ed). 2012; 4: 2558-2578.

6. Klug TL, Bast RC Jr, Niloff JM, Knapp RC, Zurawski VR Jr. Monoclonal antibody immunoradiometric assay for an antigenic determinant (CA 125) associated with human epithelial ovarian carcinomas. Cancer Res. 1984; 44: 10481053.

7. Cragun JM. Screening for ovarian cancer. Cancer Control. 2011; 18: 16-21.

8. Staab HJ, Hornung A, Anderer FA, Kieninger G. Clinical significance of the circulating tumor-associated antigen CA 19-9 in cancers of the digestive tract. Dtsch Med Wochenschr. 1984; 109: 1141-1147.

9. Lamerz R. Role of tumour markers, cytogenetics. Ann Oncol. 1999; 10 Suppl 4: 145-149.

10. Fletcher RH. Carcinoembryonic antigen. Ann Intern Med. 1986; 104: 66-73.

11. Pectasides D, Mylonakis A, Kostopoulou M, Papadopoulou M, Triantafillis D, Varthalitis J, Dimitriades M, Athanassiou A. CEA, CA 19-9, and CA-50 in monitoring gastric carcinoma. Am J Clin Oncol-Canc. 1997; 20: 348-353.

12. Yang AP, Liu J, Lei HY, Zhang QW, Zhao L, Yang GH. CA72-4 combined with CEA, CA125 and CA19-9 improves the sensitivity for the early diagnosis of gastric cancer. Clin Chim Acta. 2014; 437: 183-186.

13. Ohtsuka T, Nakafusa Y, Sato S, Kitajima Y, Tanaka M, Miyazaki K. Different roles of tumor marker monitoring after curative resections of gastric and colorectal cancers. Dig Dis Sci. 2008; 53: 1537-1543.

14. Yamao T, Kai S, Kazami A, Koizumi K, Handa T, Takemoto N, Maruyama M. Tumor markers CEA, CA199 and CA125 in monitoring of response to systemic chemotherapy in patients with advanced gastric cancer. Jpn J Clin Oncol. 1999; 29: 550-555.

15. Sisik A, Kaya M, Bas G, Basak F, Alimoglu O. CEA and CA 19-9 are Still Valuable Markers for the Prognosis of Colorectal and Gastric Cancer Patients. Asian Pac J Cancer Prev. 2013; 14: 4289-4294.

16. Shimada H, Noie T, Ohashi M, Oba K, Takahashi Y. Clinical significance of serum tumor markers for gastric cancer: a systematic review of literature by the Task Force of the Japanese Gastric Cancer Association. Gastric Cancer. 2014; 17: 26-33.

17. Tian SB, Yu JC, Kang WM, Ma ZQ, Ye X, Cao ZJ, Yan C. Combined Detection of CEA, CA 19-9, CA 242 and CA 50 in the Diagnosis and Prognosis of Resectable Gastric Cancer. Asian Pac J Cancer Prev. 2014; 15: 6295-6300.

18. Nam DH, Lee YK, Park JC, Lee H, Shin SK, Lee SK, Lee YC, Cheong JH, Hyung WJ, Noh SH, Kim CB. Prognostic value of early postoperative tumor marker response in gastric cancer. Ann Surg Oncol. 2013; 20: 3905-3911.

19. Liu X, Cai H, Wang Y. Prognostic significance of tumor markers in T4a gastric cancer. World J Surg Oncol. 2012; 10: 68 .

20. Duraker N, Celik AN. The prognostic significance of preoperative sevum CA 19-9 in patients with resectable gastric carcinoma: Comparison with CEA. J Surg Oncol. 2001; 76: 266-271.

21. Marrelli D, Roviello F, De Stefano A, Farnetani M, Garosi L, Messano A, Pinto E. Prognostic significance of CEA, CA 19-9 and CA 72-4 preoperative serum levels in gastric carcinoma. Oncology. 1999; 57: 55-62.

22. He CZ, Zhang KH, Li Q, Liu XH, Hong Y, Lv NH. Combined use of AFP, CEA, CA125 and CA19-9 improves the sensitivity for the diagnosis of gastric cancer. BMC Gastroenterol. 2013; 13: 87.

23. Sun Z, Zhang N. Clinical evaluation of CEA, CA199, CA72-4 and CA125 in gastric cancer patients with neoadjuvant chemotherapy. World J Surg Oncol. 2014; 12: 397.

24. Polat E, Duman U, Duman M, Derya Peker K, Akyuz C, Fatih Yasar N, Uzun O, Akbulut S, Birol Bostanci E, Yol $\mathrm{S}$. Preoperative serum tumor marker levels in gastric cancer. Pak J Med Sci. 2014; 30: 145-149.

25. Ishigami $\mathrm{S}$, Natsugoe $\mathrm{S}$, Hokita $\mathrm{S}$, Che $\mathrm{X}$, Tokuda $\mathrm{K}$, Nakajo A, Iwashige H, Tokushige M, Watanabe T, Takao S, Aikou T. Clinical importance of preoperative carcinoembryonic antigen and carbohydrate antigen 19-9 levels in gastric cancer. J Clin Gastroenterol. 2001; 32: 4144.

26. Li F, Li S, Wei L, Liang X, Zhang H, Liu J. The correlation 
between pre-operative serum tumor markers and lymph node metastasis in gastric cancer patients undergoing curative treatment. Biomarkers. 2013; 18: 632-637.

27. Jing JX, Xu XQ, Du LL, Tian BG, Sun T, Zhao XW, Han CZ. Clinical assessment and prognostic evaluation of tumor markers in patients with gastric cancer. Int J Biol Markers. 2013; 28: 192-200.

28. Mihmanli M, Dilege E, Demir U, Coskun H, Eroglu T, Uysalol MD. The use of tumor markers as predictors of prognosis in gastric cancer. Hepatogastroenterology. 2004; 51: 1544-1547.

29. Wobbes T, Thomas CM, Segers MF, Nagengast FM. Evaluation of seven tumor markers (CA 50, CA 19-9, CA 19-9 TruQuant, CA 72-4, CA 195, carcinoembryonic antigen, and tissue polypeptide antigen) in the pretreatment sera of patients with gastric carcinoma. Cancer. 1992; 69: 2036-2041.
30. Choi SR, Jang JS, Lee JH, Roh MH, Kim MC, Lee WS, Qureshi W. Role of serum tumor markers in monitoring for recurrence of gastric cancer following radical gastrectomy. Dig Dis Sci. 2006; 51: 2081-2086.

31. Zhou P, Qu H, Shi H, Sun G, He Q. Predictive value of CA125 in peritoneal metastasis and prognosis of patients with gastric carcinoma [Article in Chinese]. Zhonghua Wei Chang Wai Ke Za Zhi. 2014; 17: 1027-1030.

32. Tocchi A, Costa G, Lepre L, Liotta G, Mazzoni G, Cianetti A, Vannini P. The role of serum and gastric juice levels of carcinoembryonic antigen, CA19.9 and CA72.4 in patients with gastric cancer. J Cancer Res Clin Oncol. 1998; 124: 450-455.

33. Huang ZB, Zhou X, Xu J, Du YP, Zhu W, Wang J, Shu YQ, Liu P. Prognostic value of preoperative serum tumor markers in gastric cancer. World J Clin Oncol. 2014; 5: 170-176. 